\title{
The Risk Estimation of Dangerous Liquid Transport
}

\author{
M. Balatka, J. Havlíček* \\ Department of Dependability and Risk, Technical University of Liberec, Liberec, Czech Republic \\ * Corresponding author: jiri.havlicek@tul.cz
}

DOI: $10.2478 / \mathrm{v} 10158-012-0026-\mathrm{Z}$

\begin{abstract}
Transportation of liquid substances by tank vehicles on the road is an integral part of our economy. It is the petroleum products, especially fuel, that are transported in the vast majority of cases. Transportation of these hazardous liquid substances must comply with strict rules to ensure maximum transport safety. Nevertheless, there are situations where the liquid outflows outside of the car tank. The most common causes are the traffic accidents, various technical failures or human failure. Immediately following this outflow surface water and soil are threatened.

Our department develops software that is able to estimate the risks of hazardous liquids escaping into the vicinity of roads. It is a complex task involving several different disciplines. The software processes geographic data for the creation of digital terrain models. These terrain models are entered into the numerical model to calculate the spreading of a liquid. An integral part of the software is the processing of uncertainties in the environmental description which may significantly affect the calculation. Due to uncertainties the stochastic approach of evaluation was chosen. Results are expressed by statistical parameters. Software provides both qualitative and quantitative results for the liquid captured on the surface, the surface infiltration, evaporation or affecting surface waters. For example, we obtain probability maps of affected areas around transport routes, mean liquid volume leaked into a stream or the confidence interval of liquid volume infiltrating into soils.

This text describes the basic logic of software function. Specific features have been described in detail, for example in Balatka \& Havlíček (2012).
\end{abstract}

KEY WORDS: Terrain digital model, simulations of natural processes, liquid, uncertainty, spill spreading.

\section{INTRODUCTION}

Transportation of liquids by road is an integral element of the current economy. Fuel occupies the largest share of liquids transported by road. Various types of acids, hydrocarbons, including their derivatives, food preparations, etc. are transported as well. This kind of transport is provided by road tankers of many types that differ in their design, tank volume, number of chambers, additional equipment, etc.

Shippers who run a business in the field of liquids transport in our country must follow rules set out by an international agreement on the transport of dangerous goods ADR. However, road transport of fluids still means some risk. In this case we mean a leak outside of the tank volume. The most common cause of leakage may be a technical fault or accident resulting in damage of the shipping tanks. The vast majority of transported liquid substances 
are industrial products which, in the transported quantities and concentrations, can bring negative results for people and all aspects of the environment. The seriousness of leakage depends on many factors, such as the quantity of liquids, hazardousness of liquid for objects nearby the leakage, climatic conditions, etc. The most threatened receptors in the first moments of leakage are the components of the environment, such as soil or surface water. This is the main difference between the leakage of a liquid substance and a gas leak. In the case of a gas leak people are the first to be threatened. The outlined risks can be currently assessed using a numerical computer model that is being developed at the Technical University of Liberec.

\section{SPREAD OF A THIN LAYER OF LIQUID}

The leakage of a liquid substance from a tank may occur if there is a failure of valves designed for filling, draining and other purposes, or if a tank shell is disrupted. The most common cause of this adverse event is a traffic accident.

Once the liquid substance begins to flow to the surface, it creates a spill, which further spreads on the surface. Temporal evolution of the behavior of a spill depends on many factors, such as terrain morphology, the physico-chemical properties of the surface and of the liquid, climatic conditions, and the course of leakage of the liquid from the tank. The level of the spill, depending on the above-mentioned factors, frequently reaches a height ranging from a few millimeters to several centimeters.

The driving force of the fluid flow is the Earth's gravity. This acts on each particle of fluid in the direction of the surface gradient. With a very low level of a spill the dominant direction of propagation is influenced by even the smallest surface details, such as tracks, furrows in the field of agricultural machinery, rutted tracks in the meadow, etc.

Between the surface and the liquid adhesion forces also act that cause the fluid to be captured on the surface. Another reason for the capture of liquid on the surface is its roughness. An important process in the spread of a spill on a permeable surface is its infiltration. Infiltration depends on the properties of the liquid, on the properties of the surface and on the climatic conditions. For example, if grassland is frozen or saturated by rainwater, infiltration is much slower than in the opposite case where infiltration is much faster. When infiltration is slower, propagation of the spill on a surface is much greater. In addition to soil and related vegetation the liquid may also affect surface water.

\section{METHODOLOGIES FOR ESTIMATING THE EXTENT OF CONTAMINATION}

The process of the spread of a thin layer of liquid substance can be predicted using different methods. The motivation for this step may be an estimation of the extent of damage to the vicinity of the accident caused by the leakage. Some methods are described in Farrar et al. (2005), Zhiming (2006) or Yellow Book (2005). There is a wide range of methods ranging from simple screening practices to sophisticated solutions of partial differential equations. However, these methods require sufficient density and accuracy of data that describe the surface of the site concerned for producing adequate results. Such data are in practice available only in a very limited form. It should be noted that the fine surface details, such as depth of ruts in the dirt road or the density of grassland is rapidly changing. Similarly, the saturation of soil by water depends on the current weather conditions. These above-mentioned examples represent a fraction of the characteristics influenced by uncertainties that can significantly affect the spillage spreading. 
Additionally our department at the Technical University of Liberec is developing its own methodology implemented by software. It is a software package composed of several computational modules and graphic applications, which was named SPILLSIM. The main emphasis is given to processing input data uncertainties. The task is divided into two stages.

- Estimate of the extent of contamination from a point source;

- Risk assessment for a part of the transport route.

Part of the route can be seen as a line object which is composed of sufficiently small segments. Each such segment represents a potential risk of fluid leakage. In the first stage, we estimate the extent of leakage for each separate small segment of road network that can be considered to be a point source of leakage. In the next stage, we assess the risks for the road network part as a whole.

To estimate the extent of contamination from a point source is a very complicated complex problem which is divided into several mutually interpenetrating parts. These are:

- Input data describing the environment, liquid substance properties and type of leakage;

- Solving of mathematical-physical models of the liquid spread;

- Processing of uncertainties.

Risk assessment for the part of the route then constitutes the final processing of data obtained from the calculations of the point source.

\section{ENVIRONMENT DESCRIPTION}

The first necessary step in the solution of the problem is to obtain suitable data describing the environment in which we want to simulate the fluid's propagation. Input data divides into altimetry, planimetry and physico-chemical properties of the surface and the liquid substance.

Altimetry is a group of data that describe the morphology or shape of the terrain. This is the most important type of data that determines the dominant direction of liquid spread. These data are normally organized either in the form of contour lines or point grids. The SPILLSIM is able to use any resource altimetry saved as a file format for geographic information systems Esri Shapefile or any text tables stored in an ASCII text file. Sufficient density and accuracy of the data is a very important feature for the purpose of simulation.

Planimetry contains data with the occurrence of surface objects. These objects are geometrically expressed by points, lines or polygons. Points represent objects with negligible areal extent, such as solitary boulders or trees, small buildings, memorials, etc. The lines represent the linear nature of the objects. Among the most common are roads, railways, waterways, and many others. The last type of surface expression are polygon objects. Polygons describe areal formations, such as buildings, vegetation cover (e.g. forest, meadow, field), water areas, etc. The software retrieves planimetry formatted in Esri Shapefile.

For the existing calculations the ZABAGED database (ZABAGED database, 2010) was used, which falls under the administration of the Czech Office for Surveying, Mapping and Cadastre. ZABAGED is a Czech acronym for the basic basis of geographic data. It is a vector model of the territory of the Czech Republic which contains altimetry and planimetry. Altimetry can be obtained in the form of contour lines and point field with grid size $10 \times 10 \mathrm{~m}$. Accuracy of heights ranges from $1.5 \mathrm{~m}$ in flat terrain to 5 meters in very rugged wooded terrain. Topography contains more than 100 types of objects organized into layers. 
After loading of the planimetry and altimetry the data are further processed into a raster surface model. The threatened area of interest is the rectangular cutout, which is divided into equal square elements. The length of the element's side is usually chosen from 0.5 to 1 meter. Each element gets its parameters that are entered directly into the simulation calculations of liquid spread.

The first parameter assigned to the element is the identifier of the surface object occurring in the position of an element. This information is obtained directly from the planimetry. Additional data of the element is altitude. This is in addition to the altimetry also determined by planimetry. Based on the elevation a digital raster elevation model is first interpolated. The altitudes are assigned to the elements. In practice the altimetry in most cases does not cover details such as terrain morphology, such as the aforementioned rutted tracks in a dirt road, saddles and ditches formed by roads or a land surface topography in the forest. These details can be predicted just by using objects, which form small changes in the surface. Based on the planimetry it is possible to edit the altitude of elements derived from altimetry. These small properties have a major impact on the direction of the spread of a thin layer of liquid substance.

With the knowledge of the types of objects that are located in the areas of elements, we can also determine the physico-chemical properties of their surfaces, which are necessary for the simulation calculations. Besides the planimetry and altimetry in the calculation a table of values assigned to individual types of surfaces is also entered. These are parameters characterizing the interaction between the surface and the respective liquid, infiltration and evaporation. The contents of the table with these properties depend both on the type of surface and the liquid, and the particular climate state. A specific liquid substance is described by density, viscosity, and other parameters. The climate state is described by parameters such as temperature or humidity.

Altimetry, planimetry and tables of physico-chemical properties together form the input data structure, which we call vector surface model.

\section{MATHEMATICAL-PHYSICAL MODEL SOLUTION}

A mathematical-physical model is used for calculations of the time evolution behavior of a spill of leaking liquid substances. The calculation is directly based on the form of a raster model of the environment. The results of the simulation calculation are the values describing how the volume of the liquid substance develops in the individual elements over time. To each element of the raster environment model are also allocated four state variables. These values represent an immediate volume of liquid per unit area of the element. The meanings of the state variables are as follows:

- The volume of liquid trapped on the surface due to roughness and adhesion;

- The volume of fluid infiltrated from the beginning of the simulation (leak);

- The volume of liquid evaporated from the beginning of the simulation (leak);

- The volume of fluid contaminating surface water from the beginning of the simulation (leak).

In addition to the raster surface model a scenario enters into the calculation which contains the following groups of parameters:

- Determination of the locality;

- The initial and boundary conditions;

- Properties of the numerical calculation;

- Types of the desired results. 
The parameters for the determination of the locality are the material for creating a raster surface model. The initial and boundary conditions describe the position and course of leakage on the plane of the raster surface model. Leakage is modeled in such a way that on the surface of defined elements the defined volume of fluid increases in each time step. These elements form a circle with a specified radius of several units of meters. This is due to the point source nature. Features of the numerical calculation include a range of the moving calculation time step, precision limit and the simulated time of spread.

Using these scenarios we can set up six different types of results. Each result is stored in a file with a special raster format, where each element is assigned a numeric value. The meanings of the first four types of result correspond to the four aforementioned state variables. These outputs can be stored for different time moments, which are selected in a script via the step notation. As the two remaining types of result it is also possible to save the grid with altitudes and raster with object identifiers.

\section{PROCESSING UNCERTAINITIES}

The vector model of the surface is a data structure that is usually affected by the uncertainties of the input data. These uncertainties arise for various reasons.

Inaccuracies of contour lines or elevation points of altimetry can generally range from a few centimeters to several meters. The character of a thin layer spill movement is already very sensitive to the terrain asperity in order of centimeters. Uncertainties in altimetry depend primarily on how the data are obtained. The most accurate method of determining the elevation is remote sensing performed by using satellites or aerial photography based on various laser measuring methods. These methods in some cases may achieve accuracies of a few centimeters. The surface in these scales is however dynamically changing both by natural processes and human activity.

Planimetry is also affected by uncertainties. The positions and shapes of buildings, roads, waterways and vegetation cover types in the data compared to the actual state may vary from a few centimeters to tens of meters. Physico-chemical characteristics are directly dependent on the current season and weather. This affects infiltrated volume and leakage distance.

An uncertainties solution means a generalization of the described deterministic problem to a stochastic approach. All values contained in the vector surface model are not understood as strict numerical values. The stochastic approach considers these values as the most probable. In terms of probability theory, each such value describes the modus of the random variable distribution. Variances of these distributions are given by the maximum possible expected deviations. These deviations are entered common to the whole group of contours lines and object types. From the expected values and deviations densities of probability distributions are constructed. We can either set the Gaussian distribution, triangular distribution or uniform distribution, which we use when we have no idea of the expected value.

With the help of a random number generator, we can create a large number of raster surface models from the probability distribution of random variables, from which we obtain the raster results of the described state variables. In this way we get a large number of spill spread variants, which are entered into the statistical processing. This method is generally called Monte Carlo. 


\section{STATISTICAL EVALUATION}

Using the Monte Carlo calculations we obtain a set of values for each state property and an arbitrary time step for each element. These values represent the selection of some probability distribution of random variables. By statistical calculations we mean estimates of the statistical parameters of this distribution. Among the statistics that the software calculates are values of distribution and quantile functions, the mean, variance, etc. Statistical calculations are divided into two types:

- Statistical calculations for individual elements;

- Statistical calculations for whole objects.

The results of the evaluation for individual elements are the estimates of liquid relative volume statistics per unit area for the individual elements. These type of results can be graphically interpreted as the spatial distribution of the threat in the vicinity of a leak. An example is on figure 1, which shows the probability distribution of the effect on particular areas in a locality after a leakage on the road.
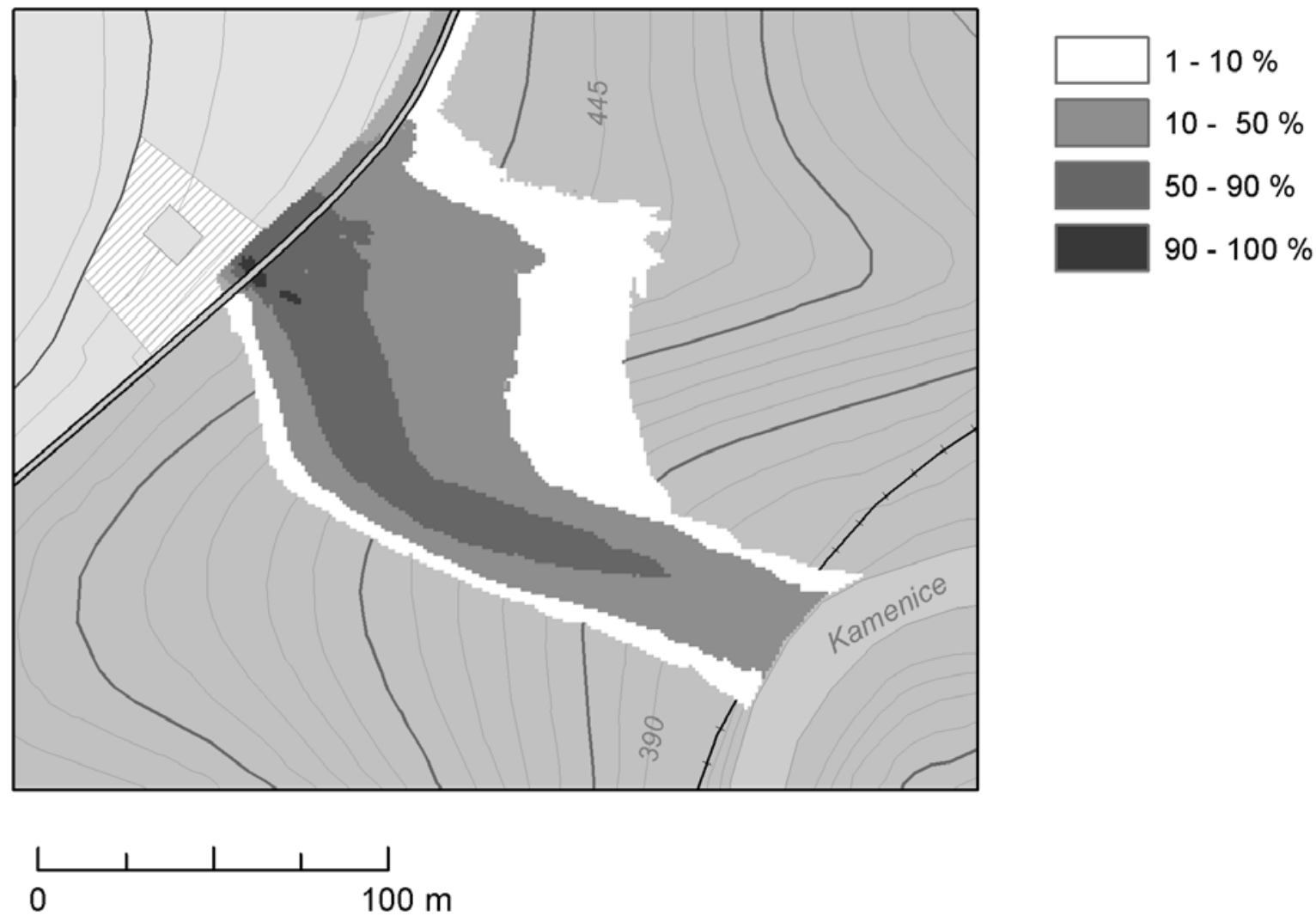

Figure 1: Probability of affection for various places of locality from a point source.

In the same way we can make estimates, for example, of mean values of infiltrated volume or the probability of exceeding the limit volume of infiltration.

The results of statistical calculations for whole objects are statistical estimates of volumes and surface extent of fluid that hit the object. A typical example is the contamination of water flows. For example, it is possible to estimate the mean values or confidence interval of fluid volume that affects a water flow in the vicinity of the road. These types of statistics can be estimated also, for example, for the area of affected grassland. 


\section{ESTIMATES OF TRANSPORT ROUTE RISKS}

Remember that the transport route can be visualized as a line consisting of sufficiently small segments, which we can consider as point sources of potential leaks. For each segment we can obtain any of the statistical parameters of one of the state variables as was mentioned before. The risk for the transport route comes from the post-processing of those results and from data on the accidents on the relevant route segment.

The data on accident rates for different road segments can be obtained from the statistics held by various institutions such as the Ministry of Transport, Police, etc. The quality of these data can be variable. Generally, it is not easy to pick out the data from only those accidents which accounted for the leak of hazardous fluids during its transport. Accident rate is described as accident frequency (unit $1 / \mathrm{km} /$ year). Threat on every position around the road is described by the frequency of affection (unit 1/year).

After evaluation we obtain a spatial distribution of frequency of affected positions around the investigated road part, as shown in the example in figure 2.

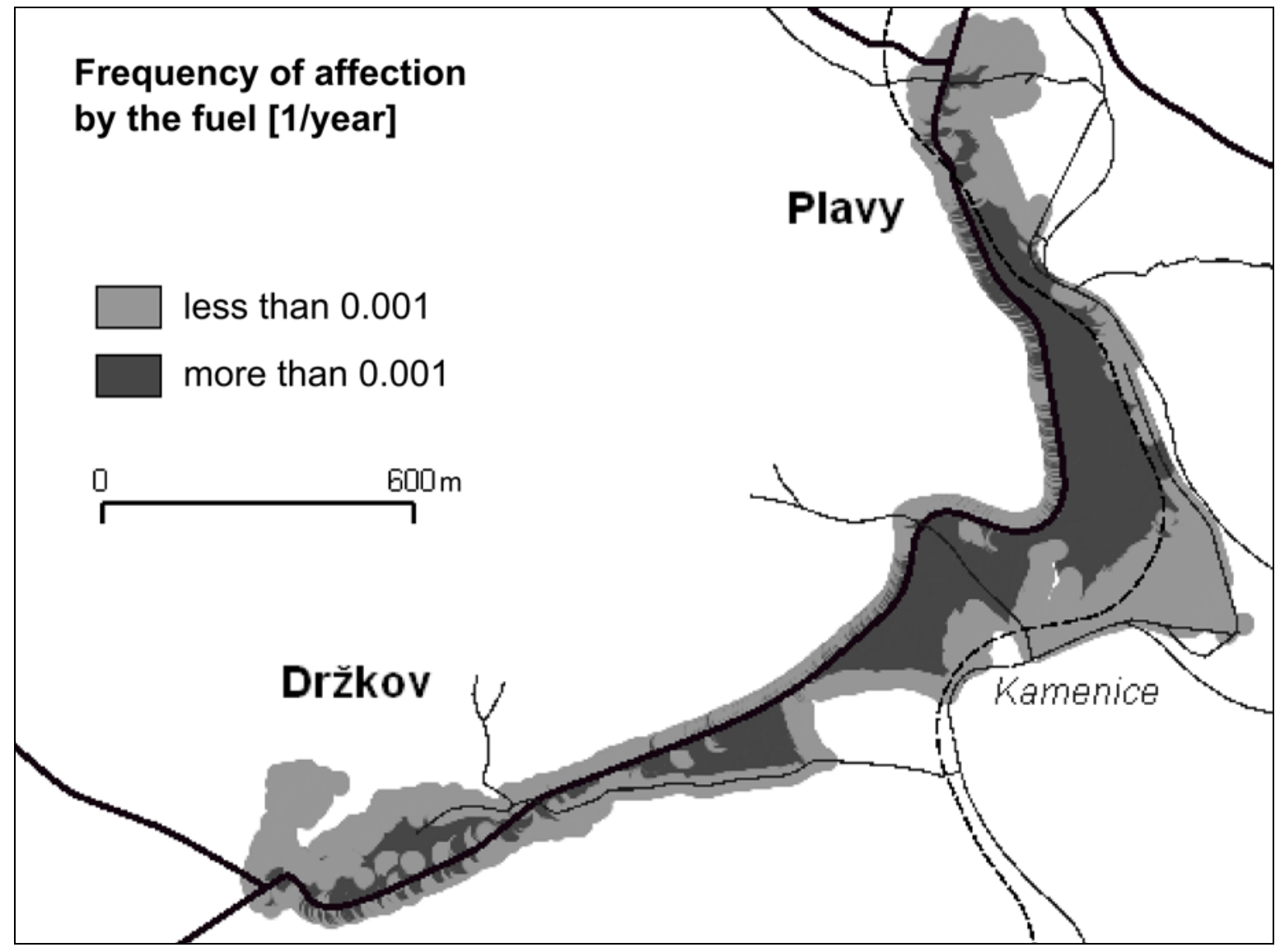

Figure 2: Example of the affected surface extent in the vicinity of the road.

\section{CONCLUSIONS}

The aim of this paper was to explain the principle function of the SIMSPILL software system, which is designed to predict the extent of the potential damage caused by the leakage of fluid transported by road. A detailed description of the model function was presented e.g. in Balatka \& Havlíček (2012). 
The current version of the system has already been successfully applied to solve two scientific research projects. The first was project no. SPII1A0/45/07 - "Complex interaction between natural processes and industry with regards to major accident prevention," supported by the Ministry of the Environment of the Czech Republic. The second was project No. 2B08011 - "Guidelines for the assessment of transport ways on biodiversity and environment components" supported by the Ministry of Education, Youth and Sports of the Czech Republic. Currently the system is used to support the project of the Technology Agency of the Czech Republic No. TA01030833 - "Integrated information system for road transportation of dangerous chemicals."

System development is still in progress and it is intended to be deployed in other areas of risk assessment.

\section{ACKNOWLEDGMENT}

This work was supported by the Technology Agency of the Czech Republic, project no. TA01030833 - Integrated information system for road transportation of dangerous chemicals.

\section{REFERENCES}

Balatka, M., Havlíček, J., 2012. Stochastical approach to spread modeling of the leaked contaminant spill. In Proceedings in Advanced Research in Scientific Areas. Žilina: EIDS - Publishing Institution of the University of Žilina, pp. 1462-1467.

Farrar, W., Galagan, Ch., Isaji, T., Knee, K., 2005. GIS technology applied to modeling oil spills on land. In ESRI international user conference.

Yellow Book, 2005. Methods for the calculation of physical effects resulting from releases of hazardous materials (liquids and gases). Third Edition, second print. CPR 14E. The Hague: Committee for the Prevention of Disasters (CPR), Directorate - General of Labour of the Ministry of Social Affairs.

Zhiming, Q., 2006. Comparison of Finite Difference Method, Philip's Method and GreenAmpt Model in Infiltration Simulation. Final project report.

ZABAGED database, 2010 [online]. (C) 2010 Český úřad zeměměřický a katastrální [Czech Office for Surveying, Mapping and Cadastre (COSMC)]. [cited 2013-04-03]. Retrieved from: http://geoportal.cuzk.cz/\%28S\%28qhez5j45ft54su554prmrf3m\%29\%29/default.aspx? mode=TextMeta\&text=dSady_zabaged\&side=zabaged\&head_tab=sekce-02-p\&menu=24 (in Czech) 